Corrigendum

\title{
Corrigendum to "Epstein-Barr Virus-Induced Gene 3 (EBI3) Blocking Leads to Induce Antitumor Cytotoxic T Lymphocyte Response and Suppress Tumor Growth in Colorectal Cancer by Bidirectional Reciprocal-Regulation STAT3 Signaling Pathway"
}

\author{
Yanfang Liang, ${ }^{1}$ Qianqian Chen, ${ }^{2}$ Wenjing Du, ${ }^{3}$ Can Chen, ${ }^{1}$ Feifei Li, ${ }^{2}$ \\ Jingying Yang, ${ }^{2}$ Jianyu Peng, ${ }^{2}$ Dongping Kang, ${ }^{1}$ Bihua Lin, ${ }^{2}$ Xingxing Chai, ${ }^{2}$ \\ Keyuan Zhou, ${ }^{2}$ and Jincheng Zeng ${ }^{2}$ \\ ${ }^{1}$ Department of Pathology, Dongguan Hospital, Medical College of Jinan University, The Fifth People's Hospital of Dongguan, \\ Dongguan 523905, China \\ ${ }^{2}$ Guangdong Provincial Key Laboratory of Medical Molecular Diagnostics, Guangdong Medical University, Dongguan 523808, China \\ ${ }^{3}$ Department of Integrative Medicine, Huashan Hospital, Fudan University, Shanghai 200040, China
}

Correspondence should be addressed to Jincheng Zeng; zengjc@gdmu.edu.cn

Received 23 November 2016; Accepted 24 November 2016; Published 27 July 2017

Copyright (C) 2017 Yanfang Liang et al. This is an open access article distributed under the Creative Commons Attribution License, which permits unrestricted use, distribution, and reproduction in any medium, provided the original work is properly cited.

In the article titled "Epstein-Barr Virus-Induced Gene 3 (EBI3) Blocking Leads to Induce Antitumor Cytotoxic T Lymphocyte Response and Suppress Tumor Growth in Colorectal Cancer by Bidirectional Reciprocal-Regulation STAT3 Signaling Pathway" [1], there were errors in the title, Introduction, Materials and Methods, and the legend of Figure 2, which should be corrected as follows:

The title should be corrected to "Epstein-Barr VirusInduced Gene 3 (EBI3) Blocking Leads to Induction of Antitumor Cytotoxic T Lymphocyte Response and Suppression of Tumor Growth in Colorectal Cancer by Bidirectional Reciprocal-Regulation STAT3 Signaling Pathway."

In the last sentence of the Introduction, "suppress" should be corrected to "promote."

In the Materials and Methods, in Section 2.3 "Balb/c" should be corrected to "BALB/c," in Section 2.4 "Balb/c" should be corrected to "BALB/c" and "RPMI1640" should be corrected to "RPMI 1640," and in Section 2.9 "1x PBS" should be corrected to " $1 \times$ PBS."

In the legend of Figure 2 "Balb/c" should be corrected to "BALB/c" and "RPMI1640" to "RPMI 1640."

\section{References}

[1] Y. Liang, Q. Chen, W. Du et al., "Epstein-Barr virus-induced gene 3 (EBI3) blocking leads to induce antitumor cytotoxic $t$ lymphocyte response and suppress tumor growth in colorectal cancer by bidirectional reciprocal-regulation STAT3 signaling pathway," Mediators of Inflammation, vol. 2016, Article ID 3214105, 11 pages, 2016. 


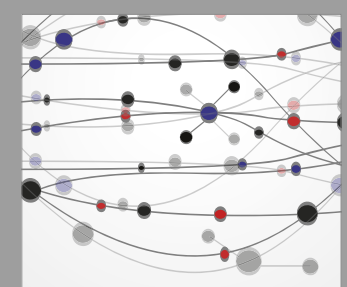

The Scientific World Journal
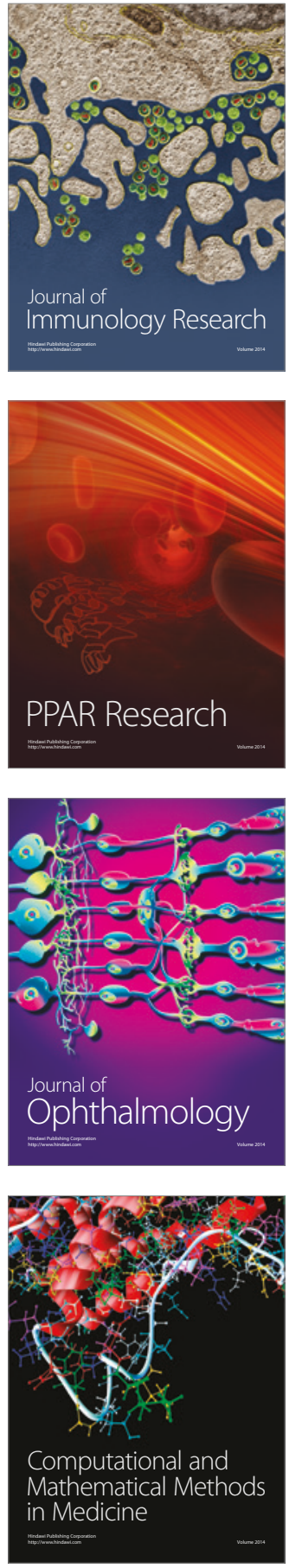

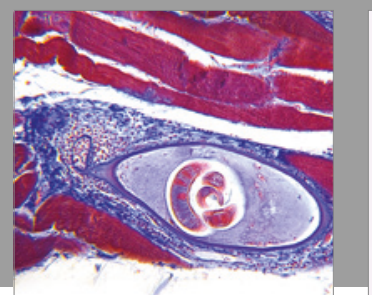

Gastroenterology Research and Practice
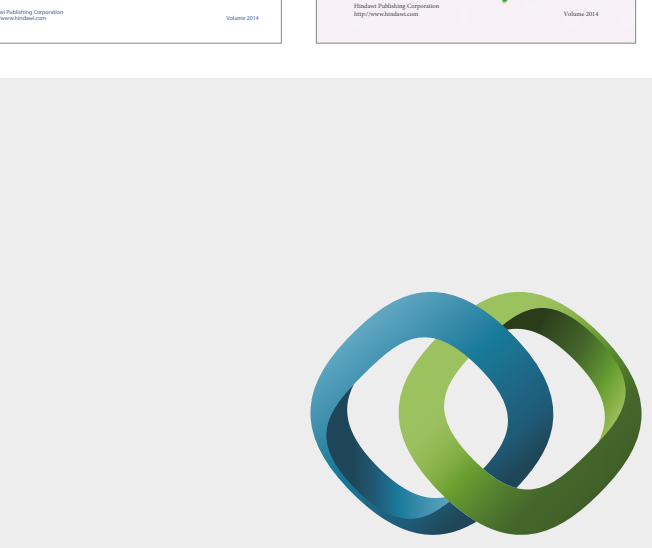

\section{Hindawi}

Submit your manuscripts at

https://www.hindawi.com
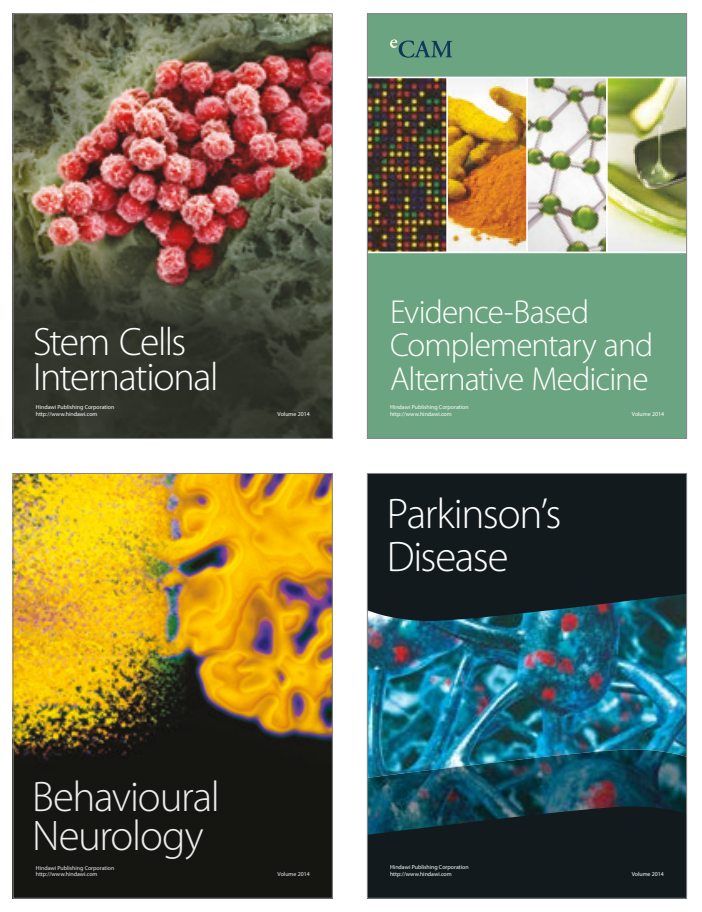
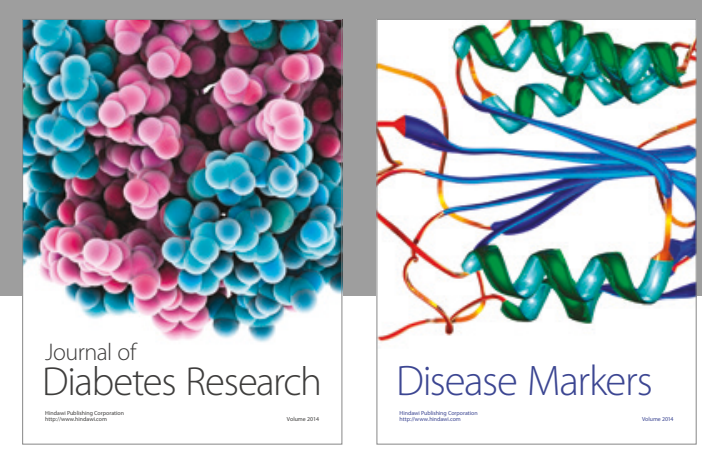

Disease Markers
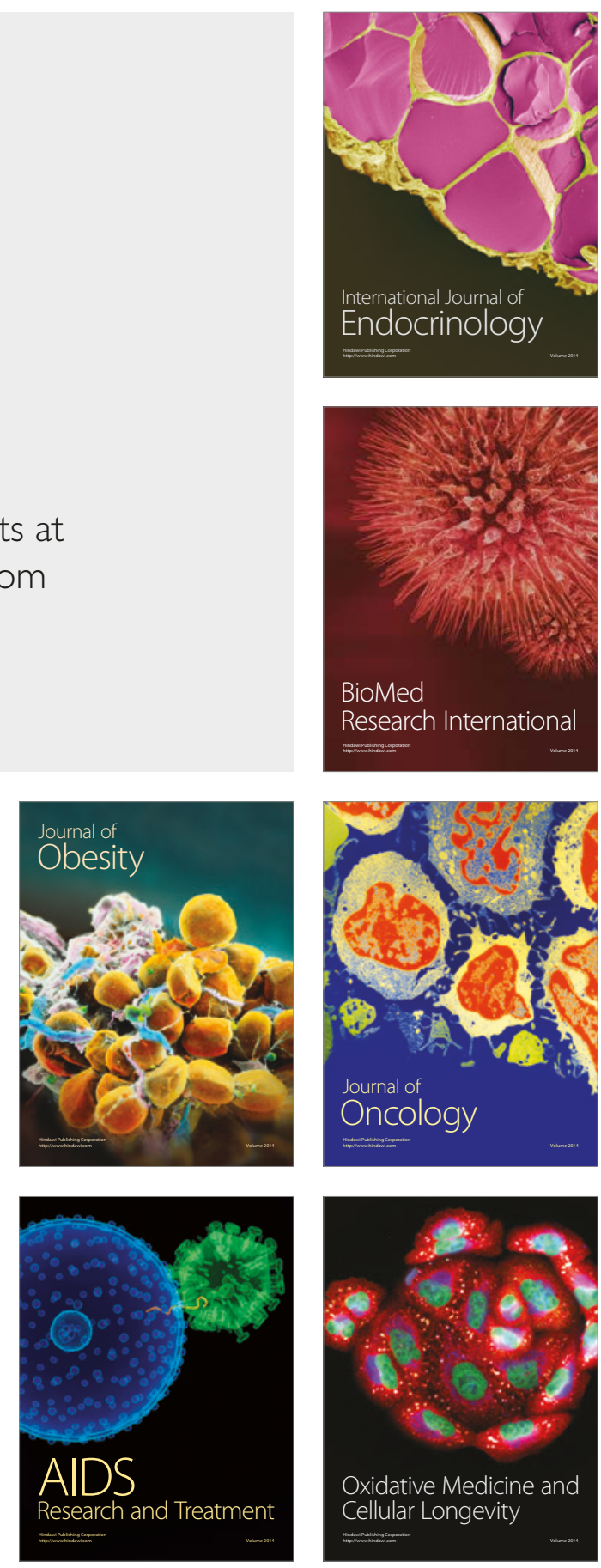